\title{
AVALIAÇÃO E GEOREFERENCIAMENTO DE ÁRVORES DE Anadenanthera peregrina (L.) Speg (Fabaceae) PARA COLETA DE SEMENTES
}

\author{
Kelmer Mozer Moro' \\ Lucimara Cruz de Souza ${ }^{2}$ \\ Adelson Lemes da Silva Júnior ${ }^{3}$ \\ Maressa Albuquerque Cortelete ${ }^{4}$ \\ Aléxia Gonçalves Pereira ${ }^{5}$ \\ Alessandra Abreu Rodrigues Vieira ${ }^{6}$ \\ Mariana Cruz De Souza ${ }^{7}$ \\ Fábio Demolinari de Miranda ${ }^{8}$
}

Resumo: A Floresta Atlântica é um dos principais biomas brasileiros e, ao longo do desenvolvimento econômico do país vem sendo antropologicamente modificada, com consequências graves para a manutenção da biodiversidade. O desmatamento é um dos principais fatores que contribuem para a perda deste bioma. Assim sendo, medidas que venham a reverter esse quadro são fundamentais. $O$ reflorestamento de áreas devastadas é uma técnica importante para reverter o esse quadro ambiental alarmante. O plantio de mudas nativas vem sendo utilizado com frequência em atividades de reflorestamento. Dentre as espécies com potencial de utilização Anadenanthera peregrina se destaca por ser pioneira e que também se mantém nas fases posteriores de sucessão ecológica. Este trabalho teve como objetivo localizar, avaliar dendrometricamente e georeferenciar árvores de A. peregrina com potencial de produção de sementes. Um total de trinta e cinco indivíduos foram localizados, avaliados e georeferenciados, sendo que todos apresentavam potencial para a produção de sementes.

Palavras-chave: Angico; Desmatamento; Reflorestamento; Sementes.

\footnotetext{
1 Graduação em Ciências Biológicas Bacharelado/Universidade Federal do Espírito Santo, Brasil. E-mail: kelmer.moro@gmail.com.

2 Pós-Graduação em Genética e Melhoramento/Universidade Federal do Espírito Santo, Brasil. E-mail: lucimaracruz15@hotmail.com.

${ }^{3}$ Universidade Federal do Espírito Santo, Brasil. E-mail: adelsonlemes@yahoo.com.br.

4 Universidade Federal do Espírito Santo, Brasil. E-mail: maressaac@yahoo.com.br.

5 Universidade Federal do Espírito Santo, Brasil. E-mail: alexiagp@gmail.com.

6 Universidade Federal do Espírito Santo, Brasil. E-mail: abreuepires@gmail.com.

7 Graduação em Agronomia/Universidade Federal do Espírito Santo, Brasil. E-mail: scruz.mariana@gmail.com.

8 Professor do Departamento de Biologia/Universidade Federal do Espírito Santo, Brasil. E-mail: fademolinari@yahoo.com.br.
} 